\title{
Erratum: Salt-Dependent Rheology and Surface Tension of Protein Condensates Using Optical Traps [Phys. Rev. Lett. 121, 258101 (2018)]
}

Louise M. Jawerth, Mahdiye Ijavi, Martine Ruer, Shambaditya Saha, Marcus Jahnel, Anthony A. Hyman,

Frank Jülicher, and Elisabeth Fischer-Friedrich

(Received 28 September 2020; published 23 November 2020)

DOI: 10.1103/PhysRevLett.125.229901

In our Letter, we have determined the surface tension $\gamma$ and the complex modulus $G^{*}(\omega)$ of protein condensates. We measured a spring constant of droplet response $\chi^{*}$ for different frequencies $\omega$ which is related to the material properties $G^{*}$ via (see our Letter and [1])

$$
\chi^{*}=\frac{\pi R\left(1-\cos \left(\theta_{0}\right)\right)}{\sum_{\substack{\ell \geq 2, \ell \text { even }}} \frac{\left(P_{\ell-1}\left(\cos \left(\theta_{0}\right)\right)-P_{\ell+1}\left(\cos \left(\theta_{0}\right)\right)\right)}{2 G^{*}(\omega) \frac{(\ell-1)\left(2 \ell^{2}+4 \ell+3\right)}{\ell(2 \ell+1)}+\frac{\gamma}{R}(\ell-1)(\ell+2)}}
$$

Here $P_{\ell}$ denotes the Legendre polynomial of degree $\ell, R$ denotes the droplet radius, and $\theta_{0}$ quantifies the size of regions where force is applied at the droplet poles. In our Letter, we used a truncation of the series (1) at $\ell=10$ which for $\theta_{0} \approx 0.1$ led to an underestimation of elastic moduli and surface tensions by roughly a factor of 2 or 1.25 , respectively. We have now numerically evaluated the series to $\ell=100$ which provides accurate values of inferred parameters. In Figs. 1(a)-1(h), we show plots with corrected data points corresponding to Figs. 3(c)-3(j) in our Letter.

Furthermore, the approximation given by Eq. (10) in our Letter for the limit $\left|G^{*}\right| R \ll \gamma$ should be improved by using [1]

$$
\gamma \approx \frac{\chi^{\prime}(\omega)}{\pi}\left(-\ln \left(\theta_{0}\right)+0.68\right) .
$$

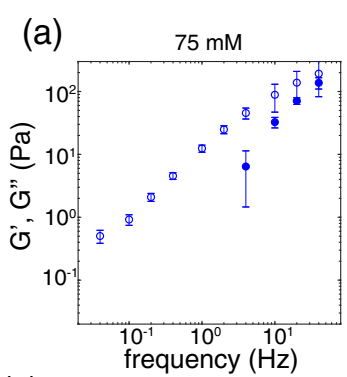

(c)

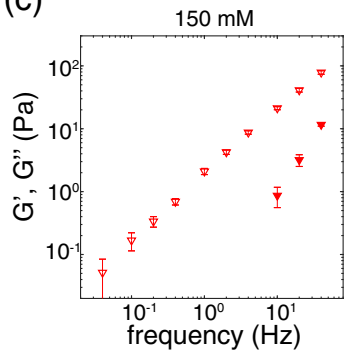

(b)

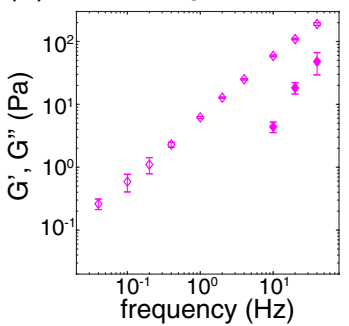

(d)

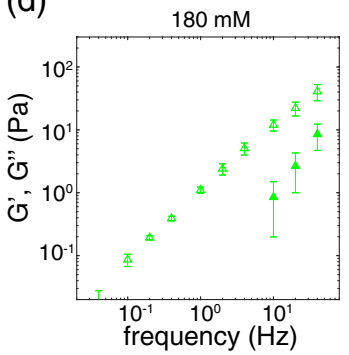

(e)

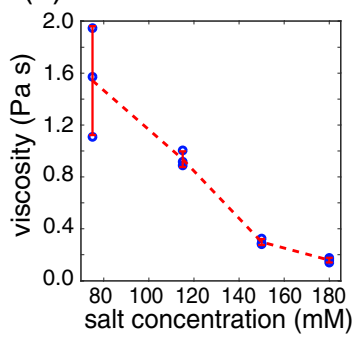

(g)

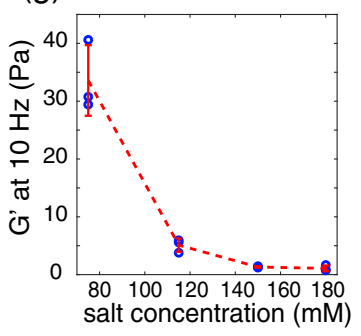

(f)

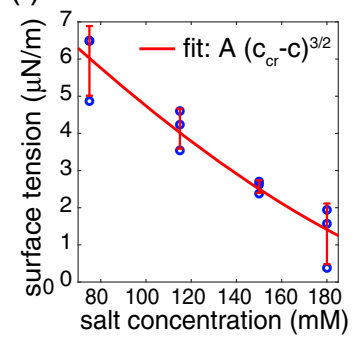

(h)

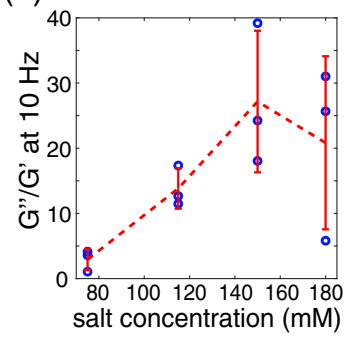

FIG. 1. Salt-dependent material properties of PGL-3 droplets. For revised data analysis, surface tension was determined from $\chi^{\prime}(\omega)$ in the low frequency regime $\left(f \leq 0.2 \mathrm{~Hz}\right.$ ) by Eq. (2). For $f \geq 4 \mathrm{~Hz}, G^{*}$ was determined numerically by inversion of $\chi^{*}(\omega)$ using Eq. (1) truncating the series after $\ell=100$. For the frequency range $f<4 \mathrm{~Hz}, G^{\prime \prime}$ was obtained by inversion of the imaginary part $\chi^{\prime \prime}(\omega)$ using Eq. (1) truncating after $\ell=100$ and setting $G^{\prime}=0$. (a)-(h) correct the panels (c)-(j) in Fig. 3 of our Letter. (f) Critical salt concentration $c_{c r}$ was fitted as $244 \mathrm{mM}$. 
The approximation Eq. (11) in our Letter for the limit $\gamma \ll\left|G^{*}\right| R$ can be improved by the following approximation:

$$
G^{*}(\omega) \approx \frac{\chi^{*}(\omega)-\left(1.25+4.36 \theta_{0}^{2}\right) \gamma}{R\left(5.47 \theta_{0}^{5}-29.28 \theta_{0}^{4}+23.29 \theta_{0}^{3}-5.08 \theta_{0}^{2}+3.79 \theta_{0}-0.02\right)} .
$$

For the experimentally relevant parameter range of $\theta_{0}$ between 0.05 and 0.3 , Eqs. (2) and (3) approximate Eq. (1) within $2 \%$ accuracy in the limit cases $G^{*}=0$ and $\gamma=0$, respectively. In the same range of $\theta_{0}$ but for finite values of $\gamma$ in the range $\gamma \leq 0.05\left|G^{*}\right| R$, Eq. (3) provides an estimate of $G^{*}$ which deviates up to $15 \%$ from the exact solution, i.e. relative errors $\Delta G^{\prime}, \Delta G^{\prime \prime}$ fulfill $\Delta G^{\prime} /|G|, \Delta G^{\prime \prime} /|G| \leq 0.15$.

We also report two typographical errors in the Supplemental Material: In Eq. (12), second line, a factor $1 / \sin (\theta)$ is missing on the right-hand side of the equation for $v_{\theta}$. In Eq. (14), $u_{r}(R, 0)$ should be replaced by $-u_{r}(R, 0)$.

[1] H. Zhou, J. Phys. Chem. B 124, 8372 (2020). 